\title{
MicroRNA-802 Inhibits Cell Proliferation and Induces Apoptosis in Human Laryngeal Cancer by Targeting cAMP-Regulated Phosphoprotein 19
}

This article was published in the following Dove Press journal: Cancer Management and Research

\author{
Huafu Ye \\ Qiaozhi Jin \\ Xiaoqiong Wang \\ Yong $\mathrm{Li}$
}

E.N.T. Department, Taizhou Municipal Hospital, Taizhou City, Zhejiang Province 318000 , People's Republic of China
Correspondence: Yong Li

E.N.T. Department, Taizhou State Hospital of Zhejiang Province, No. 38I

Zhongshan East Road, Taizhou City

318000, Zhejiang Province, People's

Republic of China

Tel +86 I5I68647760

Email prqinmo27I026@I26.com
Background/Aims: miR-802 plays a key role in cancer progression and development. The purpose of this work is to investigate the functional role of miR-802 in laryngeal cancer and to elucidate the function of miR-802 and cAMP-regulated phosphoprotein 19 (ARPP19) on laryngeal cancer.

Methods: RT-qPCR was applied to study the expression level of ARPP19 and miR-802 in the laryngeal carcinoma cell lines and tissues. CCK-8, colony formation, flow cytometry (FACS) assay were used to study the effect of ARPP19 and miR-802 on apoptosis, proliferation, and cell cycle of laryngeal carcinoma cells. Target gene prediction and luciferase reporter gene assay were applied to identify target gene of miR-802. The transcriptional mRNA and protein expression levels of ARPP19 were measured by RT-qPCR or Western blotting.

Results: miR-802 was down-regulated in laryngeal carcinoma cell lines and tissues. Laryngeal cancer cells transfected by miR-802 mimic were significantly inhibited in the terms of cell colony formation and proliferation. Furthermore, miR-802 can inhibit the expression level of ARPP19 by directly targeting the 3 ' untranslated region (3'-UTR) of ARPP19. Overexpression of the ARPP19 gene can reverse the suppressive effect of miR-802 on laryngeal cancer cells.

Conclusion: miR-802 can exert tumor suppressor effects in laryngeal carcinoma by targeting ARPP19, indicating that miR-802 protein may play a role of potential therapeutic target for clinical laryngeal cancer.

Keywords: miR-802, laryngeal carcinoma, ARPP19, proliferation, cell cycle

\section{Introduction}

Laryngeal squamous cell carcinoma (LSCC) is a popular malignant tumor in otorhinolaryngology. $^{12,17}$ It is the second-largest head and neck epithelial tumor in the world. ${ }^{24}$ Clinical randomized controlled trials for the treatment of laryngeal cancer are difficult to implement. Therefore, the current optimal treatment for laryngeal cancer is still controversial. Surgery and radiotherapy are the main treatments, and TNM staging is the main factor to consider when choosing treatment. $^{3,5}$ In the past 10 years, surgical methods of laryngeal protection have become more mature, and the use of total laryngectomy has become less. ${ }^{18,24}$ Studies have shown that the cure rate of early-stage laryngeal cancer patients is $80-90 \%$ without considering the influence of treatment methods, while the cure rate of advanced patients is only $60 \%{ }^{25}$ Therefore, the most effective treatment of laryngeal cancer is early prevention and early diagnosis. It can improve the 
prognosis of patients with laryngeal cancer, preserve the speech function of patients and boost their life quality. The inactivation of tumor inhibitory genes and the oncogenes activation are the molecular basis of cell carcinogenesis. Therefore, the treatment of laryngeal cancer by altering or modifying the related genes and their expression products has become a research hotspot in the biological treatment of laryngeal cancer. ${ }^{4,15}$

MicroRNAs (miRNAs) are a classical type of noncoding single-stranded RNA molecules with a length of approximately $22 \mathrm{nt}$ that control gene expression or not at the post-transcriptional level. ${ }^{1,6}$ MiRNA can specifically induce the degradation of target gene $\mathrm{m}$ RNA and inhibit the translation of target gene m RNA. It exerts a significant regulatory place in the process of cell development, differentiation, proliferation, and apoptosis. ${ }^{23,27}$ Currently, 500 miRNAs have been confirmed in the human genome, with more than 200 miRNAs are involved in cancer development. In recent years, miRNA has been shown to exert a significant regulatory place in the process of cancer development. ${ }^{9}$ However, the research of miRNAs is still in its infancy, its achievements in tumor gene therapy have attracted widespread attention. ${ }^{28}$ MiR-802 had a location on chromosome 21 and studies have found that overexpression of miR-802 gene can inhibit the proliferation of breast cancer cell. And it was found that miR-802 expression level was obviously reduced in GC cell lines and tissues. The strong expression level of miR-802 induces proliferation and enhanced apoptosis of GC cells. But very limited research have been carried out on the key role of miR-802 of laryngeal cancer. ${ }^{26}$

miR-802 can regulate more than 100 genes in a "one-to-many" manner, and multiple molecular pathways related to proliferation, apoptosis, differentiation, and cycle of cancer cells. Therefore, targeting miR-802 maybe more effective than traditional metastasis-related proteins in controlling multi-gene altered metastasis phenotypes. However, it is still unknown about the role that miR-802 play in the development and occurrence of laryngeal squamous cell carcinoma. We hope to identify if it is related to the invasion and metastasis of LSCC, and the pathways or molecular mechanisms that are involved. This study was to explore the influence of miR-802 on the apoptosis and proliferation of LSCC, and reveal the function of miR-802 and cAMP-regulated phosphoprotein 19 (ARPP19) on laryngeal cancer.

\section{Methods and Materials}

\section{Patient Samples and Cells}

The study was approved by the Research Ethics Committee of Taizhou State Hospital of Zhejiang Province (Taizhou, China). Informed consent was gained from all the patients. Normal tissues and postoperative laryngeal cancer tissues were selected from the patients who underwent clinical surgery at Taizhou State Hospital of Zhejiang Province. The patient consent was written informed consent, and that this was conducted in accordance with the Declaration of Helsinki. All tissue specimens were collected and promptly frozen in the container of liquid nitrogen.

\section{Cell Culture and Transfection}

HEp-2 cells (Human laryngeal squamous cell carcinoma) and SNU899 or TU212 cells (human laryngeal squamous cell carcinoma) were ordered from the Chinese Academy of Sciences (CAS). HEK293T cells were ordered from the Institute of Biochemistry and Cell Biology (IBCB, Shanghai). BEAS-2B cells (The human normal bronchial epithelial) were purchased from the American Type Culture Collection (ATCC, USA). All cells were maintained in DMEM medium (Gibco, USA) with 10\% fetal bovine serum (FBS, Invitrogen), and the cells were placed in a $5 \% \mathrm{CO}_{2}, 37^{\circ} \mathrm{C}$ incubator. When the cells reached the coverage of approximately $70 \%$, transfection was performed with a Lipofectamine 2000 kit (Invitrogen, USA) under the instructions of the manufacturer.

\section{MicroRNA (miR) and Plasmid Construction} miR-802 mimic and its negative control vector (miR-NC), siRNA against the cAMP-regulated phosphoprotein 19 (ARPP19) (si-ARPP19) and si-NC were ordered from GenePharma Company (Shanghai, China). The ARPP19 gene was introduced into the pCDNA3.1 vector to construct an ARPP19 overexpression vector. The 3'UTR region of ARPP19 with the inducible binding site of miR-802 was amplified with PCR method and then cloned into the luciferase reporter plasmid pGL3 (Promega, USA). QuikChange SiteDirected Mutagenesis Kit (Stratagene, USA) was used to bring mutations in the RNA-802 binding site of ARPP19 3'UTR, and sequencing was used to verify the success of the construct.

\section{RNA Extraction and RT-qPCR}

Total RNA was enriched from laryngeal carcinoma tissues and cells with TRIzol reagent. And we use total RNA to synthesize complementary DNA (cDNA) with the SuperScript III kit. The 
expression level of miR-802 gene was measured by LightCyclerTM 480 system, and the U6 snRNA was selected as an internal standard control.

RT-qPCR analysis of ARPP19 and the standardized control or reference gene GAPDH was applied with SYBR Premix Ex Taq kit (TaKaRa, China). The relative expression indicator of every gene was computed and normalized to the U6 snRNA or GAPDH Normalization was performed by using the $2^{-\triangle \Delta C T}$ method relative to U6-small nuclear RNA. The following primers were used for qRT-PCR:

1. miR-802-forward 5'-CGTTGTGTAGCTTATCAGA CTG-3';

2. miR-802-reverse 5'-AATGGTTGTTCTCCACACT CTC-3';

3. U6-forward 5'-CTCGCTTCGGCAGCACA-3';

4. U6-reverse 5'-aacgcttcaggaatttgcgt-3';

5. ARPP19-forward 5'-GCCTGGAGGTTCAGATTTC TTA-3';

6. ARPP 19-reverse 5'-caccagtgacctccgtcttat-3';

7. GAPDH-forward 5'-GCTGGCGCTGAGTACGTCG TGGAGT-3';

8. GAPDH-reverse 5'-CACAGTCTTCTGGGTGGCA GTGATGG-3';

\section{Cell Proliferation Assay}

Transfected laryngeal cancer cells were placed into a 96well plate with a density of $1 \times 10^{3}$ cells per well. Three wells were repeated for each group. After incubation $2 \mathrm{~h}$, $10 \mu \mathrm{L} /$ well of CCK8 reagent was added into each well. Finally, the OD value of the cell liquid was detected by an ELISA method (enzyme-linked immunosorbent assay).

\section{Colony Formation Test}

Transfected laryngeal carcinoma cells $\left(1 \times 10^{3}\right.$ cells/well $)$ were placed into a six-well plate, incubated for $24 \mathrm{hrs}$. Then, the cells were exposed to ADR for $24 \mathrm{hrs,} \mathrm{each} \mathrm{well}$ was washed, and the medium was changed. After that, culture for 8 days. After fixation with $10 \%$ formaldehyde for 40 mins, the resulting colonies were counted and stained with crystal violet $(0.1 \%)$ for 10 mins. Cell viability was plotted with the software of GraphPad Prism 6.

\section{Cell Cycle Analysis}

Cycle analysis of laryngeal cancer cells was applied with a kit of Cell Cycle Detection (Beckman Coulter, USA). The trypsin without EDTA was used to digest the transfected cells and cell collection was done by centrifugation. The collected cells were washed twice in PBS, and the transfected cells were permed in pre-cooled $70 \%$ ethanol and incubated overnight at $4{ }^{\circ} \mathrm{C}$ avoiding the light. After washed twice with $1 \mathrm{X}$ DPBS, the transfected cells were placed into $400 \mu \mathrm{L}$ of the staining solution and incubated for half an hour. The cell cycle of the transfected cells was then measured by flow cytometry (FACSort, USA) with an emission wavelength of $525 \mathrm{~nm}$ at a $525 \mathrm{~nm}$ excitation. Cell cycle changes were analyzed with ModFit 3.2 software (Beckman Coulter).

\section{Apoptosis Assay}

The transfected cells were adhered to the wall and washed two times with pre-cooled $1 \mathrm{X}$ DPBS, and the cells were mixed up in $1 \mathrm{X}$ binding buffer $(100 \mu \mathrm{L})$. Then, Annexin V FITC PI detection kit (Roche Applied Science, Germany) was used for cell staining according to the instructions. Analysis of apoptosis was applied with a flow cytometer (BD Biosciences, USA).

\section{Dual-Luciferase Reporter Gene Assay}

The 3'-UTR of the human ARPP19 gene was amplified with human liver cDNA by PCR and cloned into XhoI and NotI sites, which is located in the downstream sequence of the luciferase gene of pGL3-control vector. WT-ARPP19-3' UTR was the name of the resulting plasmid. The $3^{\prime}-\mathrm{UTR}$ was replaced by synthetic intact $3^{\prime}$-UTR DNA with errormatched seed region mutation and inserted into the pGL3control vector (Ambion) to generate a mutant construct. The pmirGLO dual-luciferase miRNA target expression vector was used in the 3 '-untranslated region (UTR) luciferase assay (Promega, USA). Hep-2 cells were placed with a density of $4 \times 10^{5}$ cells $/ \mathrm{mL}$ in 24 -well plates. After that, Lipofectamine 2000 kit was used to transfect the cells into plasmids (wild type or mutant) with mimics of microRNA802 or MICs of microRNA-NC and ARPP19. Double Luciferase Report Analysis System (Promega, USA) was used to measure the bioactivity of luciferase in fireflies and sea kidneys, and it was normalized to the activity of luciferase in sea kidneys.

\section{Western Blot}

After the total protein was cleaved using a lysis buffer containing 1\% PMSF, the concentration of protein was measured with a BCA Protein Assay Kit (Sigma, USA). The lysate was applied to a $12 \%$ polyacrylamide gel for electrophoresis experiments. After electrophoresis, it was transferred into a polyvinylidene fluoride membrane. We used 5\% milk to block the treated membranes and the members were incubated 
with ARPP19 antibody (1/1000 dilution; Santa Cruz Biotechnologies, USA) overnight at $4^{\circ} \mathrm{C}$ temperature. Then, it was incubated with anti-rabbit IgG poly-antibody $(1 / 6000$ dilution; GE) for $2 \mathrm{hrs}$ at $37^{\circ} \mathrm{C}$. GAPDH antibody (1/3000 dilution; Bioworld) was utilized as a control standard. All protein bands were detected using an ECL Western blotting kit (Amersham Biosciences, UK).

\section{Statistical Method}

The monitoring data were analyzed by SPSS19.0 statistical software. The data analysis results were expressed as mean \pm standard deviation (mean $\pm \mathrm{SD}$ ). The data analysis between the two groups was applied by the $t$-test. The data analysis between multiple groups was based on oneway variance analysis (ANOVA). The LSD test was used for subsequent analysis, $\mathrm{P}<0.05$ showed that the difference between two groups was statistically significant.

\section{Results}

\section{miR-802 Was Down-Regulated in Laryngeal Cancer Cell Lines and Tissues}

In order to assess the biological role of miR-802 gene in the progression of laryngeal carcinoma, RT-qPCR was used to measure gene expression of miR-802 in 38 pairs of laryngeal carcinoma tissue specimens and their matched adjacent noncancerous laryngeal tissues. Our data indicated that the expression level of miR-802 gene was significantly decreased in laryngeal carcinoma tissues with a comparison to that in the nearby non-cancerous laryngeal carcinoma tissues $(\mathrm{P}<0.01)$ (Figure 1A and B). Next, the expression level of the miR-802 gene in the cell lines of laryngeal carcinoma (TU212, Hep-2, and SNU899) and the cell lines of human normal bronchial epithelial (BEAS-2B) were further analyzed. As indicated in Figure $1 C$, the expression of miR-802 gene was obviously decreased in the laryngeal cancer cell line with a comparison to that in the BEAS-2B cell lines $(\mathrm{P}<0.01)$. In the 3 laryngeal carcinoma cell lines, the expression level of miR-802 gene was the lowest in cell line of Hep-2, so Hep-2 cell was chosen for subsequent studies. These data suggested that miR-802 gene may be related to the development of laryngeal cancer.

\section{miR-802 Inhibited Cell Proliferation Laryngeal Cancer Cells}

In order to further investigate the role of miR-802 gene in laryngeal cancer, $100 \mathrm{nM}$ of miR-802 or miR-NC plasmid vector was utilized to transfect tumor cells of Hep-2 cells and TU212 Hep-2 cells. The data were indicated in
Figures 2A and 6A. MiR-802 expression levels were obviously enhanced in miR-802 mimic-transfected Hep-2 cells and TU212 Hep-2 cells with a comparison to cells transfected by miR-NC $(\mathrm{P}<0.01)$.

After confirmed successful transfection, the influence of the miR-802 gene on Hep-2 cell growth, colony formation, apoptosis, and cell cycle were analyzed. The CCK-8 proliferation assay indicated an obvious reduce the proliferation rate of miR-802 mimic-transfected Hep-2 cells with a comparison to cells transfected by miR-NC vector $(\mathrm{P}<0.01)$ (Figure 2B). Colony formation results showed that miR-802 mimic-transfected Hep-2 cells obviously reduced the number of cell colonies $(\mathrm{P}<0.01)$ with a comparison to cells transfected by miR-NC vector (Figure 2C). FACS data indicated that contrasted to miR$\mathrm{NC}$ transfected tumor cells, the cell ratio of G0/G1 phase of Hep-2 tumor cells with miR-802 mimics transfection was obviously improved, and the percentage of tumor cells in $\mathrm{S}$ phase were obvious reduced $(\mathrm{P}<0.01)$ (Figure 2D). In addition, it was shown that the apoptotic rate was obviously improved in miR-802 mimic-transfected Hep-2 cells with a comparison to that in miR-NC transfected tumor cells $(\mathrm{P}<0.01)$ (Figure $2 \mathrm{E}$ ). These data showed that miR-802 overexpression suppressed proliferation of laryngeal carcinoma cells, induced apoptosis, and caused G0/G1 phase cell arrest in cells.

\section{ARPPI9 Was a Direct Target Protein of miR-802 in Laryngeal Cancer Cells}

In order to determine the potential mechanism of action of miR-802 gene in laryngeal cancer, it was predicted that ARPP19 was treated as a potential target for miR-802 by three miRNA databases (PITA, miRmap, and microT) (Figure 3A). Then, whether miR-802 can directly bind to ARPP19 was investigated, WT or MT ARPP19-3'-UTR was cloned and luciferase bioactivity was measured. As indicated in Figure 3B, miR-802 gene overexpression significantly decreased luciferase bioactivity of WT ARPP19-3'UTR vector $(\mathrm{P}<0.01)$; however, there was no significant variation in luciferase bioactivity of MT ARPP19-3'UTR (Figure 3B).

In order to further study the influence of miR-802 gene on ARPP19, ARPP19 mRNA and protein expression levels in Hep-2 cells with miR-NC and miR-802 mimic transfection were determined. The data indicated that ARPP19 mRNA and protein expression was obviously reduced in Hep-2 tumor cells with miR-802 transfection with comparison to miR-NC transfected cells $(\mathrm{P}<0.01)$ 



Figure I miR-802 was down-regulated in laryngeal cancer cell lines and tissues. (A) The expression yield of miR-802 gene in 38 pairs of laryngeal cancer tissues and their pair-matched nearby noncancerous laryngeal tissues. (B) The expression yield of miR-802 gene in laryngeal cancer tissues and pair-matched nearby noncancerous laryngeal tissues. (C) The expression yield of miR-802 in three laryngeal cancer cell lines and BEAS-2B cells. **P $<0.01, * P<0.05$.

(Figure 3C and D). In addition, it was indicated that protein and mRNA levels of the ARPP19 gene were significantly up-regulated in the three laryngeal carcinoma cell lines with comparison to BEAS-2B tumor cells $(\mathrm{P}<0.05, \mathrm{P}<0.01)$ (Figure $3 \mathrm{E}$ and $\mathrm{F})$. And the expression level of ARPP19 was significantly raised in laryngeal carcinoma tissues with a comparison to that in the nearby non-cancerous laryngeal carcinoma tissues $(\mathrm{P}<0.01)$ (Figure 3G). All the data indicated that ARPP19 was a direct target protein of miR-802 in laryngeal cancer cells

\section{ARPPI 9 Silencing Inhibited Cell Growth} and Induced Apoptosis in Laryngeal

\section{Cancer Cells}

In order to analyze the role of ARPP19 down-regulation, the expression of ARPP19 in laryngeal carcinoma cells by si-ARPP19 or si-NC transfection was silenced. The data indicated that protein and mRNA levels of ARPP19 gene were significantly decreased in Hep-2 cells with siARPP19 transfection with a comparison to that in the 
A

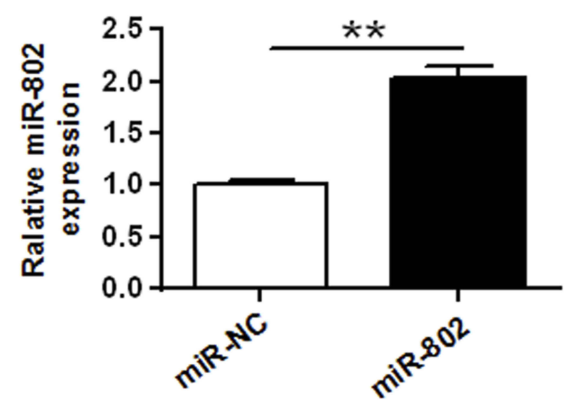

C

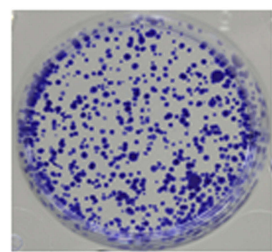

miR-NC

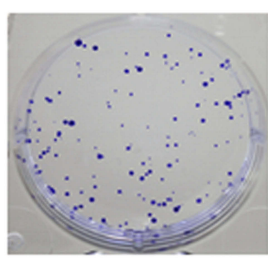

miR-802
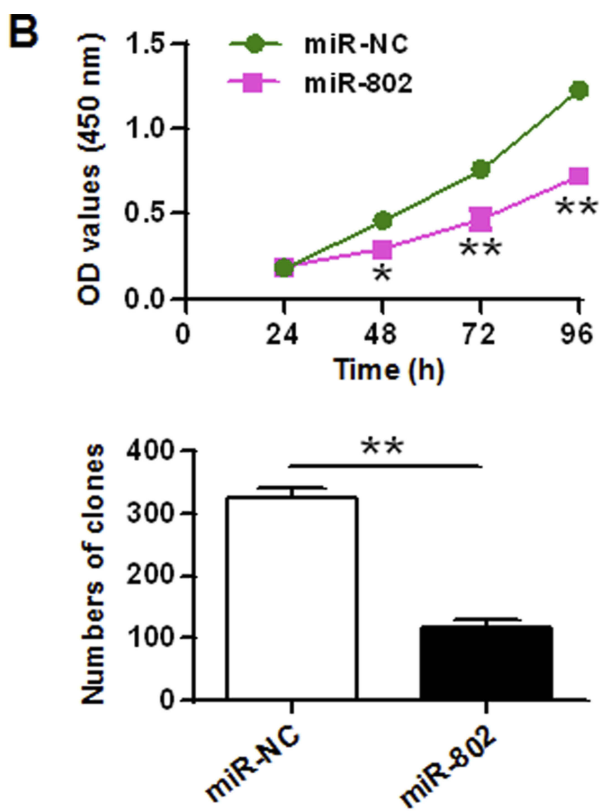

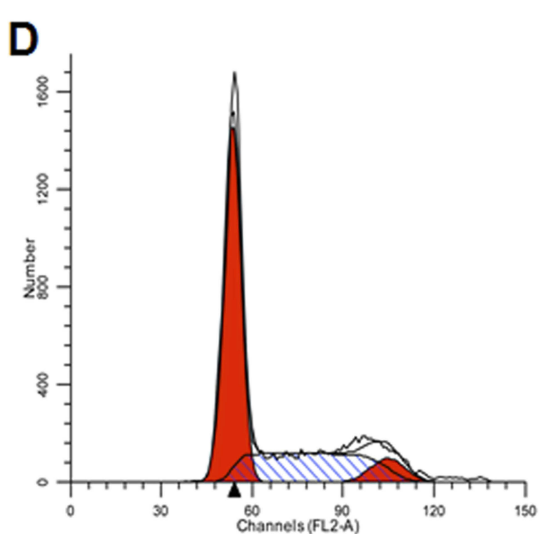

E

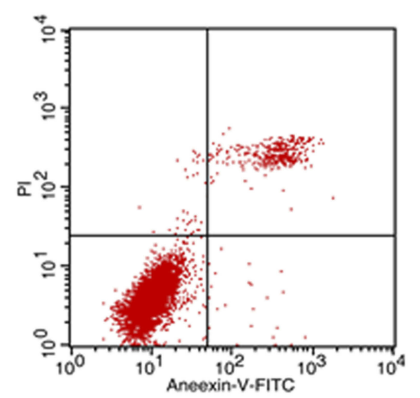

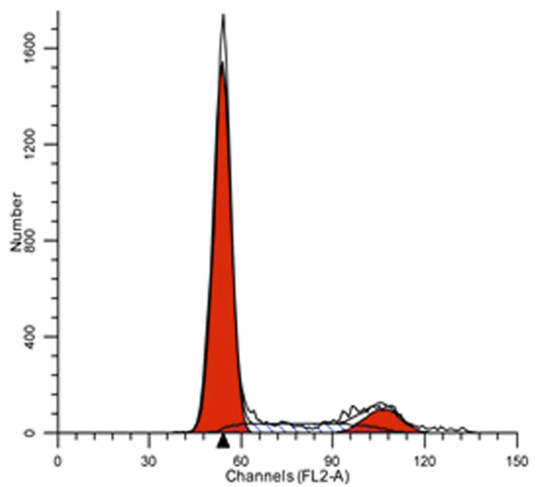

miR-802

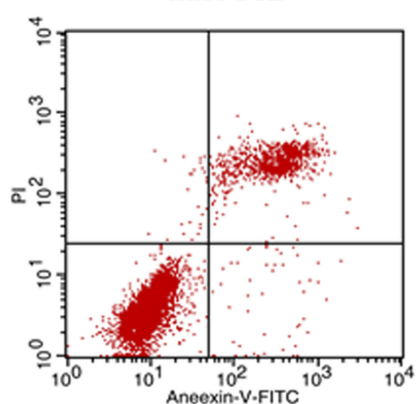

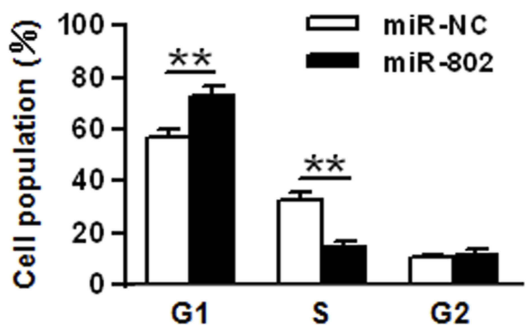

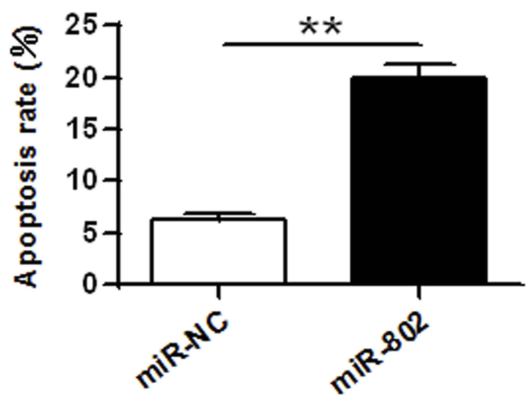

Figure 2 MiR-802 inhibited cell growth and induced apoptosis in laryngeal cancer cells. (A) The expression level of the miR-802 gene in Hep-2 tumor cells with miR-NC and miR-802 mimic transfection. (B) Cell growth in Hep-2 tumor cells with miR-NC and miR-802 mimic transfection. (C) Colony formation in Hep-2 tumor cells with miR-NC and miR-802 mimic transfection. (D) Cell cycle stage in Hep-2 tumor cells with miR-NC and miR-802 mimic transfection. (E) Apoptosis in Hep-2 cells $t$ with miR-NC and miR-802 mimic transfection. ${ }^{*} * \mathrm{P}<0.01, * \mathrm{P}<0.05$.

control part $(\mathrm{P}<0.01)$ (Figure $4 \mathrm{~A}$ and $\mathrm{B})$. In addition, CCK-8 proliferation assay indicated that the proliferation rate of Hep-2 cells with si-ARPP19 transfection was obviously lower than the proliferation rate of the control part $(\mathrm{P}<0.01)$ (Figure 4C). Colony formation data indicated that the number of Hep-2 cell colonies transfected with si-ARPP19 was significantly reduced with a comparison to that in the control group $(\mathrm{P}<0.01)$ (Figure 4D). Flow cytometry data indicated that contrasted to the control group, the percentage of cells arrested in the G0/G1 phase of Hep-2 cells transfected with si-ARPP19 was obviously enhanced, and the percentage of cells of the 
A

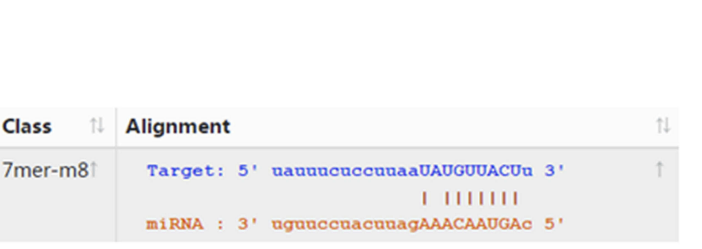

B

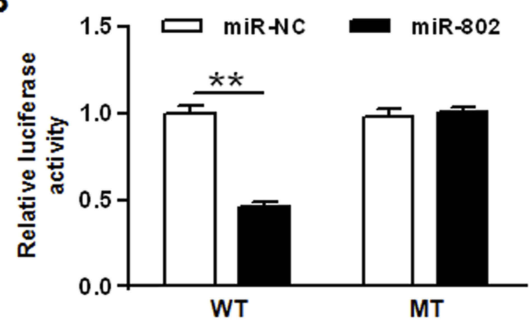

C

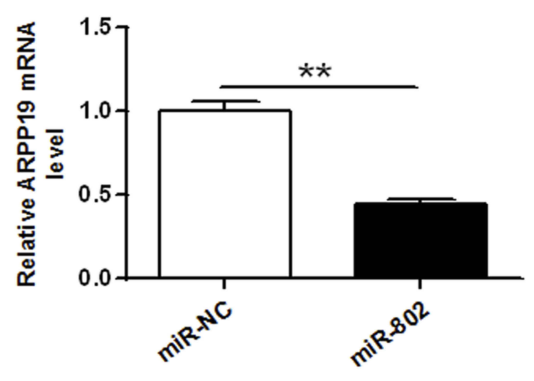

$\mathbf{F}$

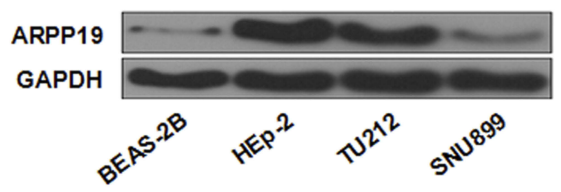

D
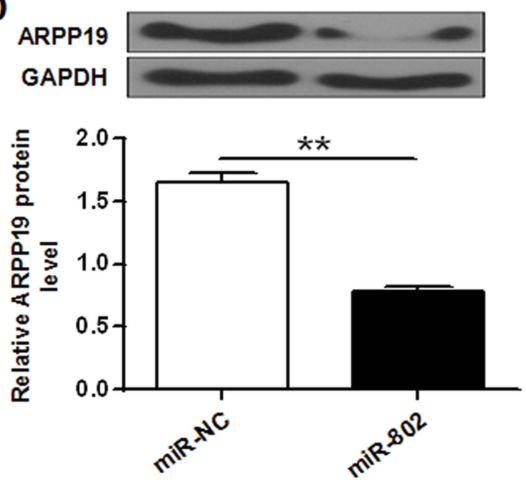

G

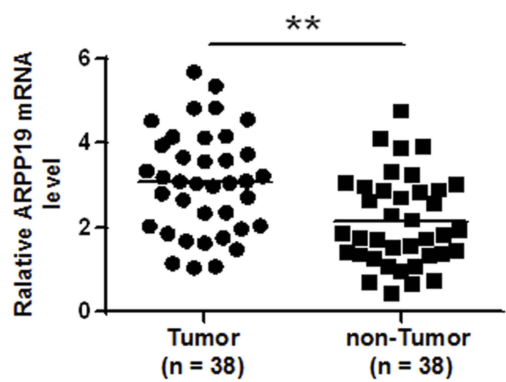

E

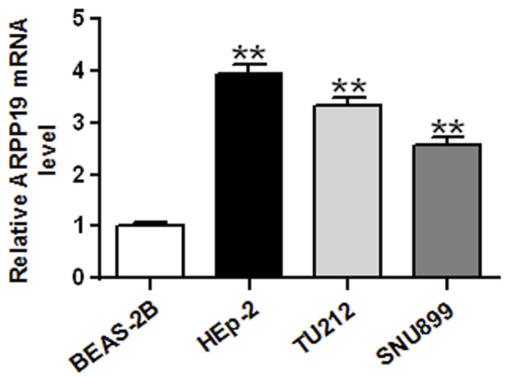

Figure 3 ARPPI9 was a direct target protein of the miR-802 gene in laryngeal cancer cells. (A) Predicted miR-802 target sequences in ARPPI9-3-UTR. (B) Relative luciferase bioactivities in Hep-2 tumor cells co-transfected with ARPPI9 3'UTR (MT or WT) reporter gene plasmid, miR-NC or miR-802 mimic. **P < 0.0I vs miR-NC. (C) ARPPI 9 mRNA expression level in Hep-2 tumor cells with miR-NC and miR-802 mimic transfection. **P < 0.01 vs miR-NC. (D) ARPPI9 expression in Hep-2 cells with miR-NC and miR-802 mimic transfection. (E) ARPPI 9 expression on the mRNA transcriptional level of BEAS-2B cells and three laryngeal cancer cell lines. **P < 0.01 vs miR-NC. (F) ARPPI 9 expression at the protein level of BEAS-2B cells and three laryngeal cancer cell lines. $* * P<0.01$ vs BEAS-2B cells. (G) The expression level of ARPPI 9 was significantly raised in laryngeal carcinoma tissues. $* * \mathrm{P}<0.0 \mathrm{I}$ vs TUMOR.

$S$ phase was obviously reduced $(\mathrm{P}<0.01)$ (Figure 4E). Besides, it was found that the apoptosis rate of Hep-2 tumor cells with si-ARPP19 transfection was obviously enhanced with a comparison to that in the control part ( $\mathrm{P}$ $<0.01$ ) (Figure 4F), and it showed similar function of the inhibitory effect of miR-802 gene overexpression on Hep2 cells.

\section{Overexpression of ARPPI9 Reversed the Effect of miR-802 in Laryngeal Cancer Cells}

In order to further study whether the biological function of the miR-802 gene in laryngeal carcinoma was regulated by ARPP19, ARPP19 expression was restored by transient transfection with the ARPP19 overexpression plasmid pCDNA3.1-ARPP19 in miR-802 mock-transfected Hep-2 cells and TU212 cells. As shown in Figures 5A, B and $6 \mathrm{~B}, \mathrm{C}$, the ARPP19 protein and mRNA levels in the miR802 treated group was obviously lower than that in the control part, and the ARPP19 mRNA and protein levels in the miR-802+ ARPP19 group were obviously elevated with a comparison to that in the miR-802 treated group $(\mathrm{P}<0.01)$.

In addition, the CCK- 8 proliferation assay indicated that the growth rate of Hep-2 tumor cells and TU212 cells in the miR-802 transfected group was obviously lower than that in the control part (Figures 5C and 6D). Colony formation data indicated that the number of cell colonies in the miR-802 transfected group was obviously lower $(\mathrm{P}<0.01)$ with a comparison to that in the control 
A

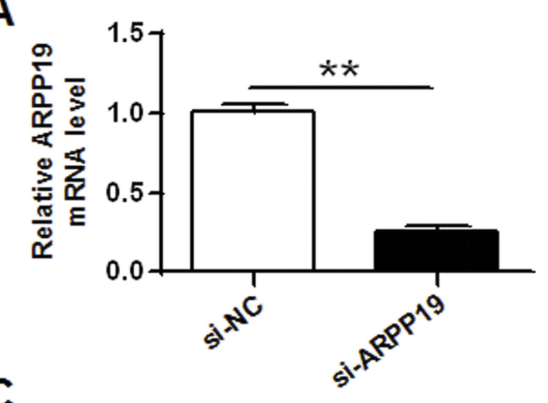

C

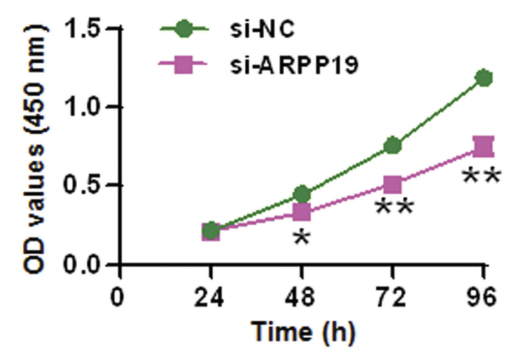

$\mathbf{E}$

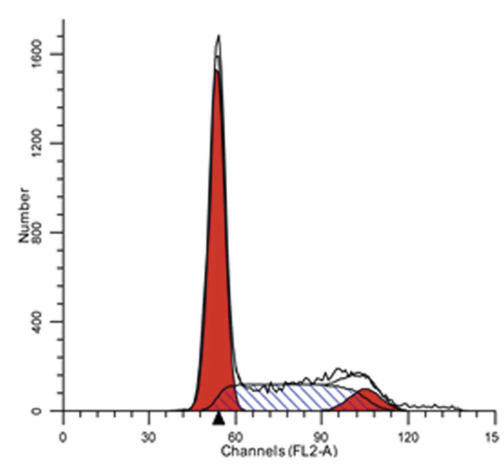

$\mathbf{F}$

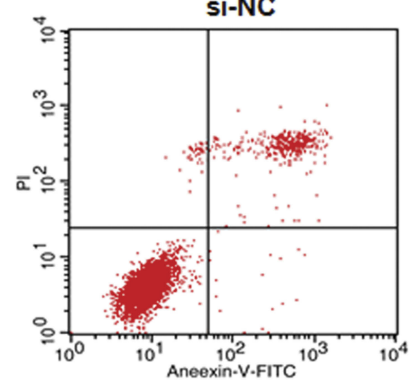

B

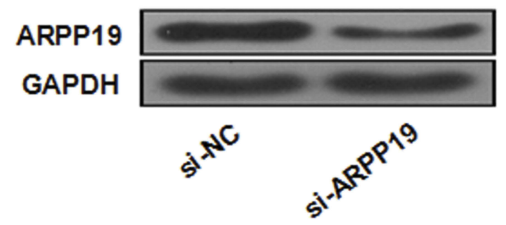

D
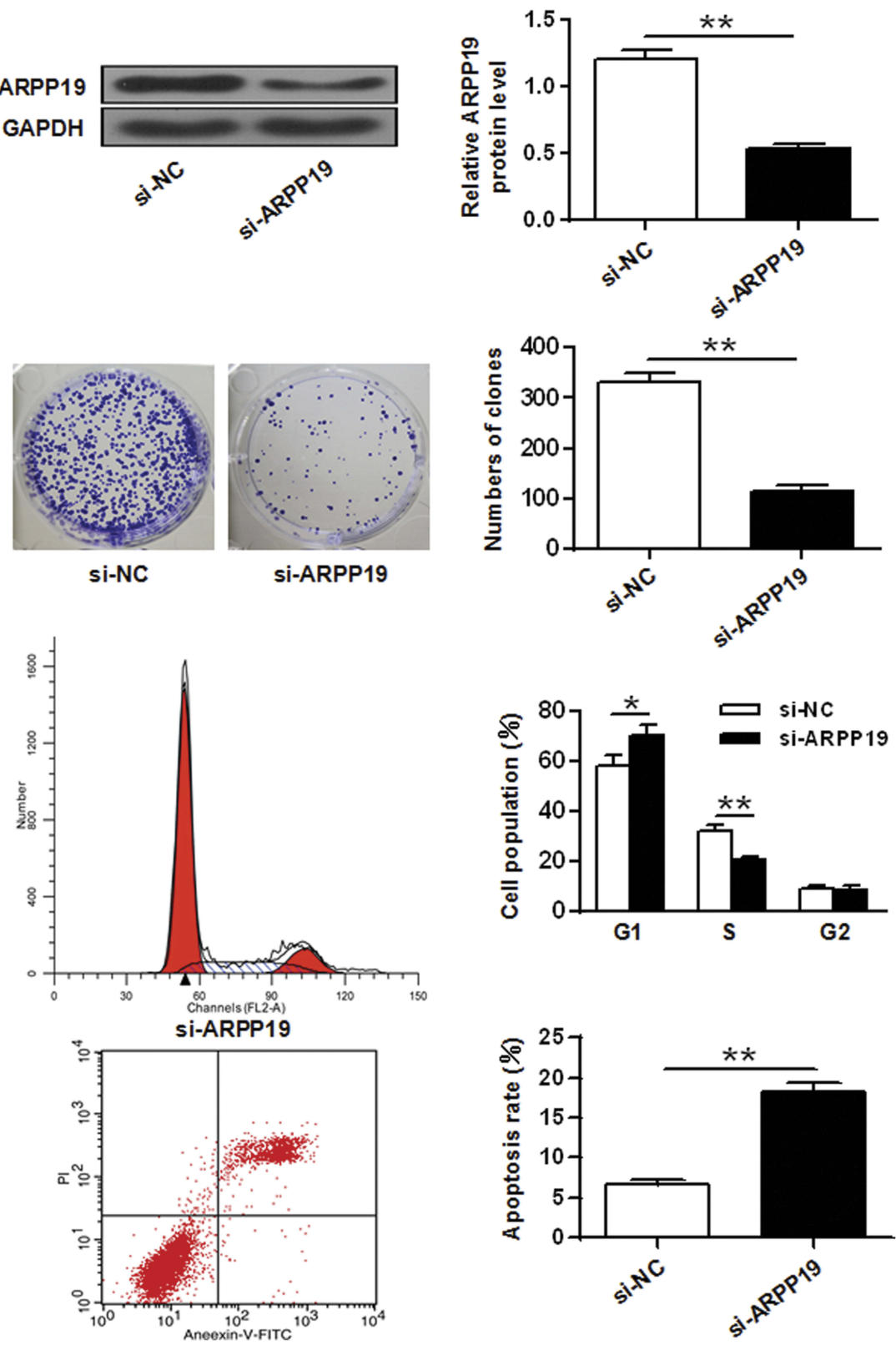

Figure 4 ARPPI 9 silencing inhibited cell growth and elicits apoptosis in laryngeal cancer cells. (A) ARPPI 9 expression on mRNA level was measured in Hep-2 tumor cells with si-NC or si-ARPPI 9 transfection. (B) ARPPI 9 gene expression at the protein level in Hep-2 tumor cells with si-NC or si-ARPPI 9 transfection. (C) Cell growth in Hep2 tumor cells with si-NC or si-ARPPI 9 transfection. (D) Colony formation in Hep-2 tumor cells with si-NC or si-ARPPI 9 transfection. (E) Cell cycle stage in Hep-2 tumor cells with si-NC or si-ARPPI 9 transfection. (F) Apoptosis was assessed in Hep-2 tumor cells with si-NC or si-ARPPI 9 transfection. $* * P<0.0$, $* P<0.05$.

part (Figure 5D). Flow cytometry data indicated that compared with the control part, the percentage of G0/G1 phase cells in the miR-802 transfected group was obviously reduced, and the percentage of cells in the $\mathrm{S}$ phase was obviously reduced $(\mathrm{P}<0.01)$ (Figure 5E). In addition, it was indicated that the apoptotic rate of miR-802 transfected group was obviously elevated with a comparison to the control part $(\mathrm{P}<0.01$ ) (Figures $5 \mathrm{~F}$ and $6 \mathrm{E}$ ), and overexpression of ARPP19 could eliminate the effects of
microRNA-802 on cell growth, colony formation, cell apoptosis, and cell cycle arrest in Hep-2 cells $(\mathrm{P}<0.01$, $P<0.05)$. All the data suggested that the miR-802 gene probably has biological effects in laryngeal cancer cells by binding to ARPP19.

\section{Discussion}

Laryngeal cancer is a popular malignant tumor in the head and neck. ${ }^{7,21}$ The pathological type is most common in 
A

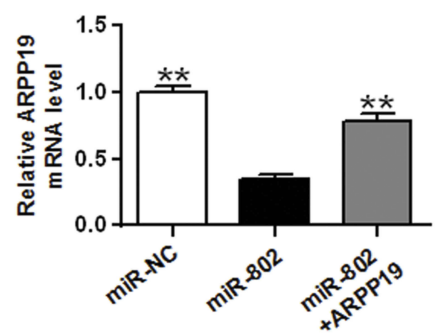

C

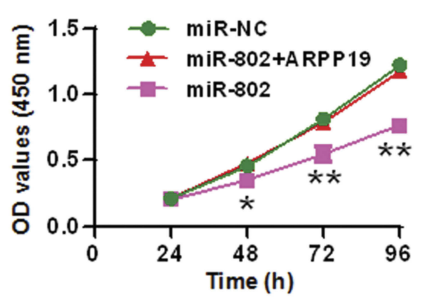

D

B

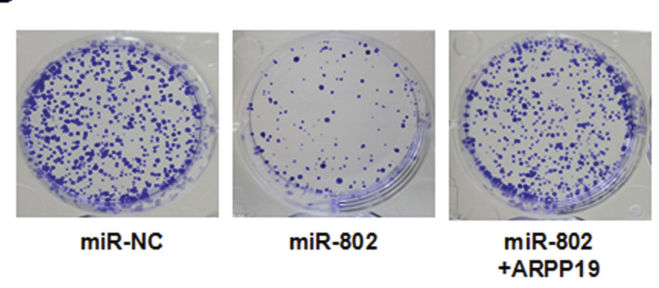

ARPP19
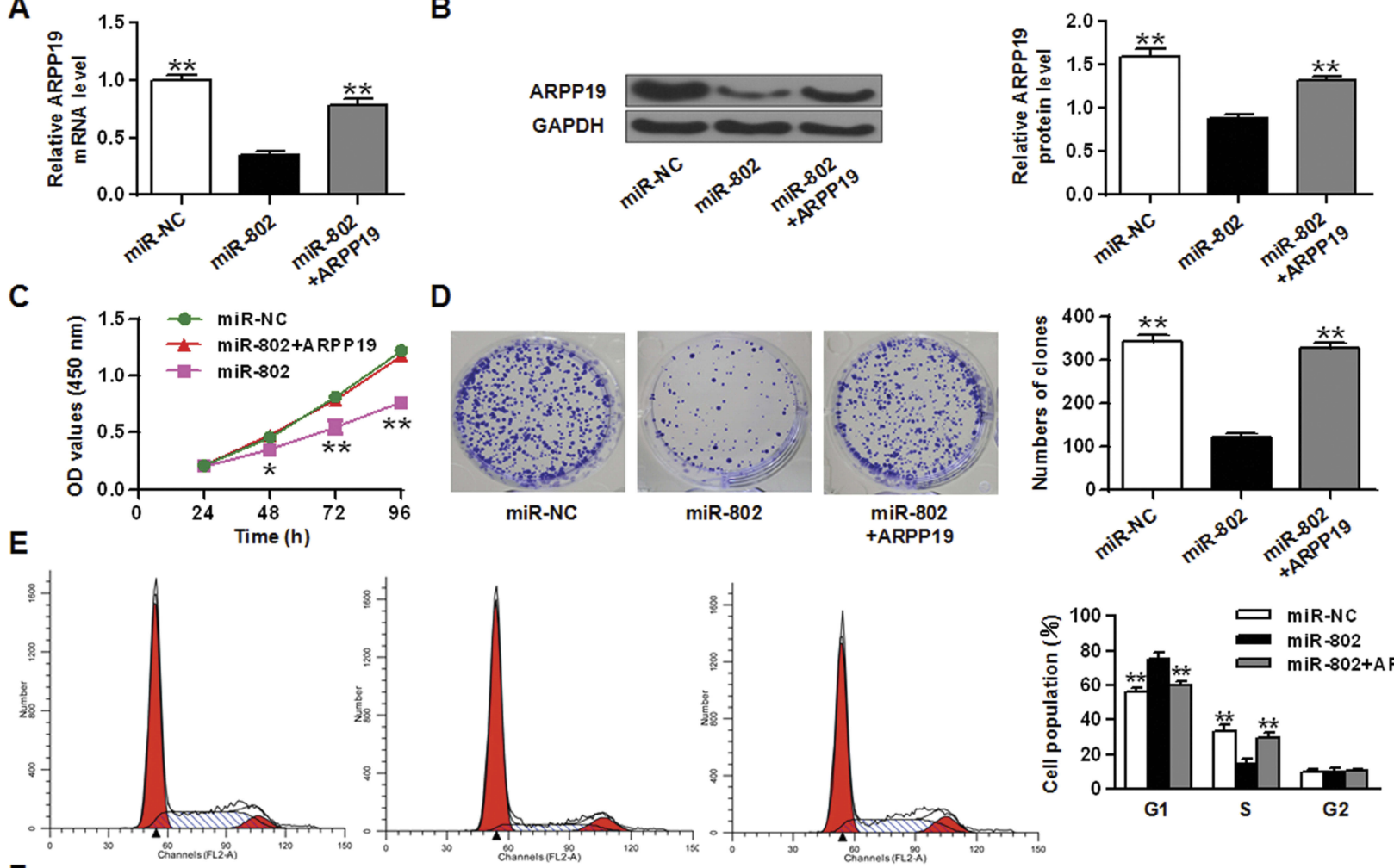

$\mathbf{F}$
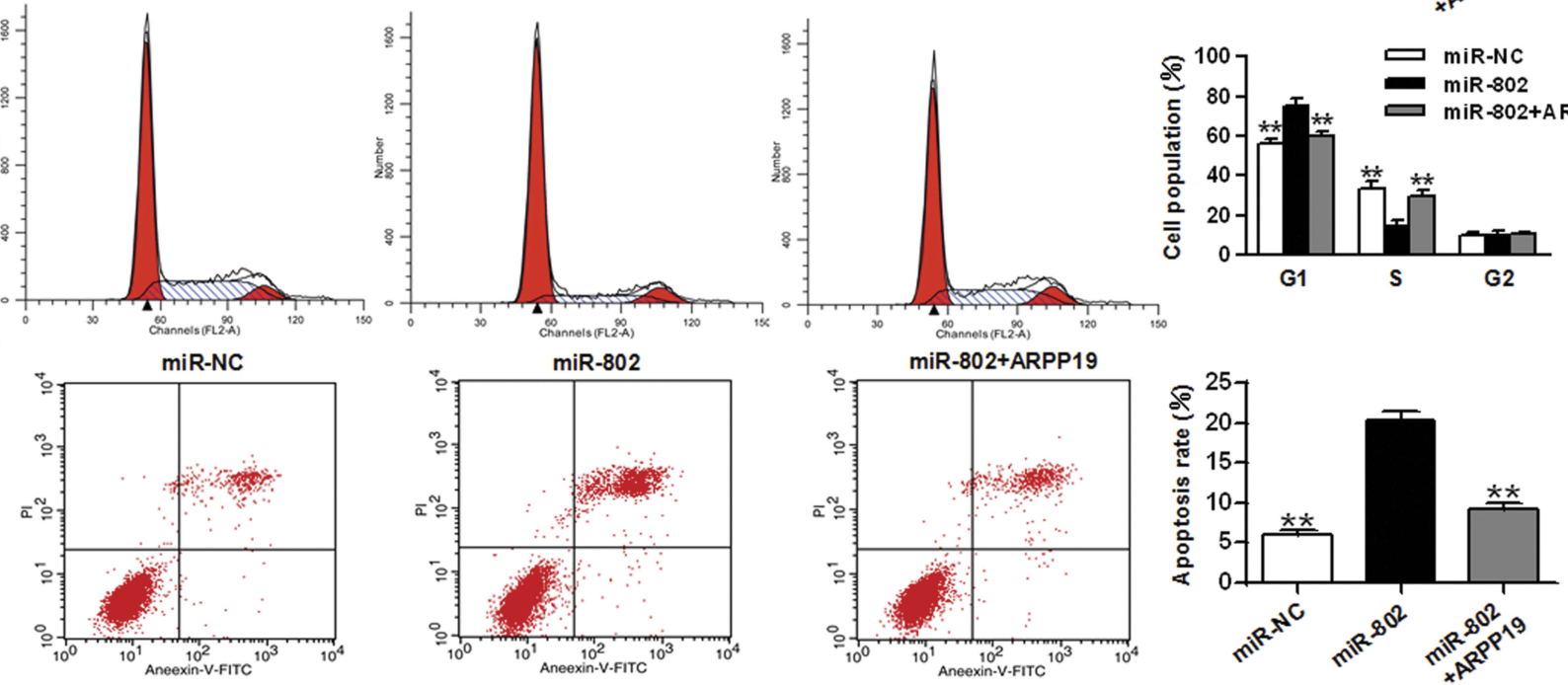

Figure 5 Overexpression of the ARPPI 9 gene reversed the influence of miR-802 in Hep-2 tumor cells. (A) ARPPI9 expression on the mRNA level in Hep-2 tumor cells with miR-NC or miR-802 mimic transfection, and with pCDNA3.I-ARPPI9 plasmid or not. (B) ARPPI9 expression at the protein level in Hep-2 tumor cells with miR-NC or miR-802 mimic transfection, and with PCDNA3.I-ARPPI9 plasmid or not. (C) Cell proliferation in Hep-2 tumor cells with miR-NC or miR-802 mimic transfection, and with PCDNA3.I-ARPPI9 plasmid or not. (D) Colony formation in Hep-2 tumor cells with miR-NC or miR-802 mimic transfection, and with pCDNA3.I-ARPPI9 plasmid or not. (E) Cell cycle stage in Hep-2 tumor cells with miR-NC or miR-802 mimic transfection, and with pCDNA3.I-ARPPI9 plasmid or not. (F) Apoptosis was detected in Hep-2 tumor cells with miR-NC or miR-802 mimic transfection, and with PCDNA3.I-ARPPI9 plasmid or not. **P $<0.01, * \mathrm{P}<0.05$.

squamous cell carcinoma, and the number of new cases accounts for $1 \%$ of all new cases of malignant tumors worldwide. ${ }^{11}$ Although the treatment methods have been continuously improved, the 5-year survival rate of cancer patients with advanced-stage has not been fundamentally elevated. Therefore, early determination and early diagnosis and treatment of laryngeal cancer metastasis are the keys to improve the survival rate of cancer patients with advanced stage.

In order to detect laryngeal cancer and improve its therapeutic effect and prognosis, it is necessary to further investigate the mechanism of action of laryngeal cancer development. Previous work has indicated that miRNA exerts a significant role in the development and occurrence of laryngeal cancer. ${ }^{20,22}$ MicroRNA (miRNA) is a type of endogenous non-coding small RNA of 18 to 25 nucleotides in length. ${ }^{19}$ MiRNA is highly conserved in evolution and has post-transcriptional gene regulatory functions. MiRNA regulates the expression of about one-third of the genes in the human genome. ${ }^{10}$ More and more studies have shown that miRNA with carcinogenic or tumor suppressor activity is related to the development and occurrence of different tumors including lung cancer, liver cancer, colon cancer, breast cancer, brain tumor, and leukemia. ${ }^{13,29}$ To date, research on the relationship between miRNA function and cancer formation for the 
A
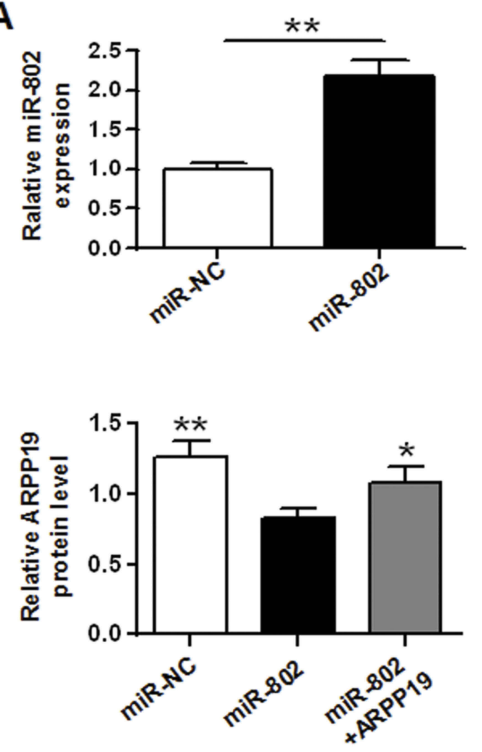

B

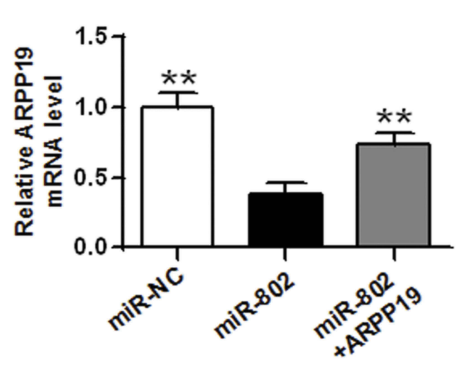

D

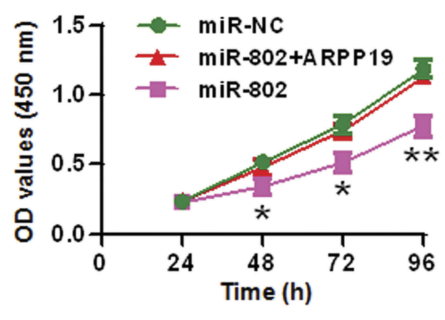

C

ARPP19 GAPDH

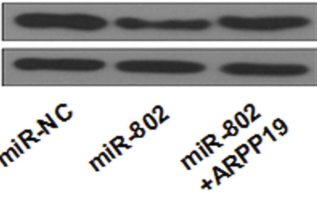

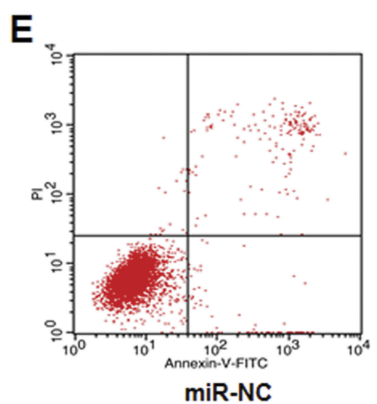
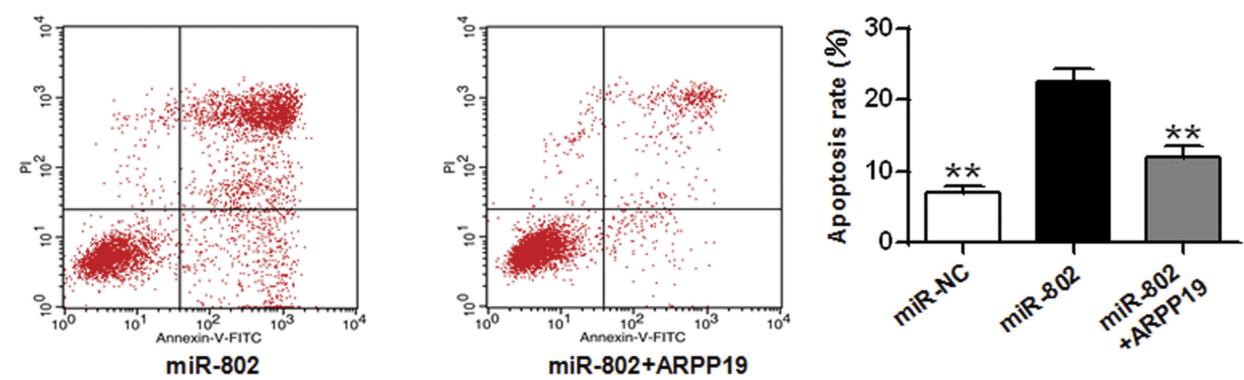

Figure 6 Overexpression of the ARPPI9 gene reversed the influence of miR-802 in TU212 tumor cells. (A) ARPPI9 expression on the mRNA level in TU2 12 tumor cells with miR-NC or miR-802 mimic transfection, and with PCDNA3.I-ARPPI9 plasmid or not. (B) ARPPI9 expression at the protein level in Hep-2 tumor cells with miR-NC or miR-802 mimic transfection, and with PCDNA3.I-ARPPI9 plasmid or not. (C) Cell proliferation in TU2I 2 tumor cells with miR-NC or miR-802 mimic transfection, and with PCDNA3.I-ARPPI 9 plasmid or not. (D) Colony formation in TU2I 2 tumor cells with miR-NC or miR-802 mimic transfection, and with pCDNA3.I-ARPPI9 plasmid or not. (E) Cell cycle stage in TU2I2 tumor cells with miR-NC or miR-802 mimic transfection, and with pCDNA3.I-ARPPI9 plasmid or not. (F) Apoptosis was detected in TU2I 2 tumor cells with miR-NC or miR-802 mimic transfection, and with PCDNA3. I-ARPPI9 plasmid or not. **P $<0.01$, *P $<0.05$.

purpose of treating cancer has become one of the hot topics at home and abroad. Previous studies found that miR-802 expression showed reduced level breast cancer cells and tissues, while overexpression of miR-802 inhibited breast cancer cell growth by suppressing FOXM1 expression. ${ }^{26}$ This study found that the expression level of miR-802 in cancer cells and tissues was significantly lower than expression level in normal adjacent normal cells and normal tissues $(\mathrm{P}<0.05)$. And miR-802 overexpression can inhibit cell viability, induce apoptosis and cause G0/G1 phase arrest in cells. In conclusion, miR802 can be treated as a potential molecular therapeutic target for laryngeal cancer, and its expression can be inhibited to control the development of laryngeal cancer.

cAMP (Cyclic Adenosine monophosphate) is a cyclic nucleotide and is an abbreviation for 'adenosine-3', 5'cyclized monophosphate". It is formed by the condensation of two phosphoric acids by adenosine triphosphate (ATP). ${ }^{14}$ AMP is an important gene expression regulator. In prokaryotic organisms, cAMP is considered to directly activate RNA polymerase to promote transcription, i.e. to promote InRNA transcription by phosphorylation of the enzyme's six factors. ${ }^{2}$ New research suggests that the role of cAMP in eukaryotic cells is associated with regulation of transcription factors. ${ }^{16}$ Phosphoprotein 19 (ARPP19) is a key gene in cAMP regulation. Studies have found that cAMP-regulated phosphoprotein 19 (ARPP19) is a target protein of mir-26a, and mir-26a can suppress cell growth by regulating ARPP $19 .{ }^{8}$ We screened ARPP19 for the target gene of miR- 802 by database. And mir802 regulated its expression level by binding to the 3'UTR of the ARPP19 gene. The ARPP19 levels in Hep-2 tumor cells with miR-802 transfection were obviously lower than cells with miR-NC transfection. In addition, ARPP19 mRNA and protein levels were obviously higher in the three laryngeal 
cancer cell lines than in BEAS-2B cells. These data suggested that ARPP19 protein was a direct target protein of miR-802 drug in laryngeal cancer cells. To further study the role of ARPP19 down-regulation, it was showed that ARPP19 levels in Hep-2 tumor cells transfected with si-ARPP19 were obviously lower than that in control part. Besides, ARPP19 silencing obviously suppressed cell growth and colony formation, cell apoptosis, elicited cell cycle arrest in G0 or G1 phase. Overexpression level of ARPP19 gene reversed the role of miR-802-induced cell growth, colony formation, apoptosis, cycle arrest in Hep-2 cells. These results suggested that miR802 may have biological effects in laryngeal cancer cells by binding ARPP19.

\section{Conclusion}

MiR-802 suppressed the proliferation of laryngeal carcinoma cells and elicited apoptosis by regulating ARPP19, indicating that miR-802 has a potential role for laryngeal cancer. It provided an experimental basis for clinical prognosis judgment and further targeted interventional therapy of this tumor.

\section{Data Sharing Statement}

The analyzed data sets generated during the study are available from the corresponding author on reasonable request.

\section{Disclosure}

The authors declare that they have no competing interests in this work.

\section{References}

1. Baek D, Villén J, Shin C, Camargo FD, Gygi SP, Bartel DP. The impact of microRNAs on protein output. Nature. 2008;455(7209):64. doi: $10.1038 /$ nature 07242

2. Bourtchuladze R, Frenguelli B, Blendy J, Cioffi D, Schutz G, Silva AJ. Deficient long-term memory in mice with a targeted mutation of the cAMP-responsive element-binding protein. Cell. 1994;79(1):59-68. doi:10.1016/0092-8674(94)90400-6

3. Cata JP, Zafereo M, Villarreal J, et al. Intraoperative opioids use for laryngeal squamous cell carcinoma surgery and recurrence: a retrospective study. J Clin Anesth. 2015;27(8):672-679. doi:10.1016/j.jclinane.2015. 07.012

4. Ciesielska U, Zatonski T, Nowinska K, et al. Expression of cell cycle-related proteins p16, p27 and Ki-67 proliferating marker in laryngeal squamous cell carcinomas and in laryngeal papillomas. Anticancer Res. 2017;37(5):2407. doi:10.21873/anticanres

5. Dirix P, Lambrecht M, Nuyts S. Radiotherapy for laryngeal squamous cell carcinoma: current standards. Expert Rev Anticancer Ther. 2010;10(9):1461. doi:10.1586/era. 10.110

6. Fabbri M, Croce CM, Calin GA. MicroRNAs. Cancer J. 2007;14 (1):759-774.

7. Facs HTHM, Porter K, Karnell LH, et al. Laryngeal cancer in the United States: changes in demographics patterns of care, and survival. Laryngoscope. 2010;116(S111):1-13.
8. Gong Y, Wu W, Zou X, Liu F, Wei T, Zhu J. MiR-26a inhibits thyroid cancer cell proliferation by targeting ARPP19. Am J Cancer Res. 2018;8(6):1030-1039.

9. Kloosterman WP, Plasterk RH. The diverse functions of microRNAs in animal development and disease. Dev Cell. 2006;11(4):441-450. doi:10.1016/j.devcel.2006.09.009

10. Lai X, Wang M, Mcelyea SD, Sherman S, House M, Korc M. A microRNA signature in circulating exosomes is superior to exosomal glypican-1 levels for diagnosing pancreatic cancer. Cancer Lett. 2017;393:86. doi:10.1016/j.canlet.2017.02.019

11. Lara PC, Cuyás JM. The role of squamous cell carcinoma antigen in the management of laryngeal and hypopharyngeal cancer. Cancer. 2015;76(5):758-764. doi:10.1002/1097-0142(19950901)76:5<758:: AID-CNCR2820760508>3.0.CO;2-9

12. Li D, Feng J, Wu T, et al. Long intergenic noncoding RNA HOTAIR is overexpressed and regulates PTEN methylation in laryngeal squamous cell carcinoma. Am J Pathol. 2013;182(1):64-70. doi:10.1016/j. ajpath.2012.08.042

13. Li Y, Chen Y, Li J, et al. Co-delivery of microRNA-21 antisense oligonucleotides and Gemcitabine using nanomedicine for pancreatic cancer therapy. Cancer Sci. 2017;108(7):1493-1503. doi:10.1111/ cas.2017.108.issue-7

14. Liu L, Das S, Losert W, Parent CA. mTORC2 regulates neutrophil chemotaxis in a cAMP- and RhoA-dependent fashion. Dev Cell. 2010;19(6):845-857. doi:10.1016/j.devcel.2010.11.004

15. Liu Y, Liu J, Wang L, Yang X, Liu X. MicroRNA-195 inhibits cell proliferation, migration and invasion in laryngeal squamous cell carcinoma by targeting ROCK1. Mol Med Rep. 2017;16(5):7154. doi: $10.3892 / \mathrm{mmr} .2017 .7460$

16. Nesterova MV, Glukhov AI, Severin ES. Effect of the regulatory subunit of cAMP-dependent protein kinase on the genetic activity of eukaryotic cells. Mol Cel Biochem. 1982;49(1):53-61. doi:10.1007/ BF00230996

17. Oktem F, Yazicilar O, Güvenç MG, et al. Urinary N-acetyl-beta -D-glucosaminidase levels in patients with laryngeal squamous cell carcinoma. J Otolaryngol. 2007;36(36):233-239. doi:10.2310/7070. 2007.0028

18. Ou XU, Xiaoming LI, Wang J, Wang J, Shen Y, Otorhinolaryngology DO. The impact of hypoxia improvement on laryngeal squamous cell carcinoma chemotherapy. J Clin Otorhinolaryngol Head Neck Surg. 2015;29 (9):824.

19. Rupaimoole R, Slack FJ. MicroRNA therapeutics: towards a new era for the management of cancer and other diseases. Nature Rev Drug Discovery. 2017;16(3):203. doi:10.1038/nrd.2016.246

20. Saito K, Inagaki K, Kamimoto T, et al. MicroRNA-196a is a putative diagnostic biomarker and therapeutic target for laryngeal cancer. PLoS One. 2013;8(8):e71480. doi:10.1371/journal.pone.0071480

21. Silver CE, Beitler JJ, Shaha AR, Rinaldo A, Ferlito A. Current trends in initial management of laryngeal cancer: the declining use of open surgery. Head Neck. 2009;266(9):1333-1352.

22. Sun X, Liu B, Zhao XD, Wang LY, Ji WY. MicroRNA-221 accelerates the proliferation of laryngeal cancer cell line Hep-2 by suppressing Apaf-1. Oncol Rep. 2015;33(3):1221-1226. doi:10.3892/or.2015.3714

23. Tavazoie SF, Alaren C, Oskarsson T, et al. Endogenous human microRNAs that suppress breast cancer metastasis. Nature. 2008;451(7175):147-152. doi:10.1038/nature06487

24. Trask DK, Wolf GT, Bradford CR, et al. Expression of Bcl-2 family proteins in advanced laryngeal squamous cell carcinoma: correlation with response to chemotherapy and organ preservation. Laryngoscope. 2010;112(4):638-644. doi:10.1097/00005537-200204000-00009

25. Wang J, Wu Y, Gao W, et al. Identification and characterization of CD133 + CD44+cancer stem cells from human laryngeal squamous cell carcinoma cell lines. $J$ Cancer. 2017;8(3):497-506. doi:10.7150/jca.17444

26. Yuan F, Wang W. MicroRNA-802 suppresses breast cancer proliferation through downregulation of FoxM1. Mol Med Rep. 2015;12 (3):4647. doi:10.3892/mmr.2015.3921 
27. Zhang B, Pan X, Cobb GP, Anderson TA. microRNAs as oncogenes and tumor suppressors. $N$ Engl J Med. 2007;302(1):1-12.

28. Zhang L, Huang J, Yang N, et al. microRNAs exhibit high frequency genomic alterations in human cancer. Proc Natl Acad Sci U S A. 2006;103(24):9136-9141. doi:10.1073/pnas.0508889103
29. Zhou F, Nie L, Feng D, Guo S, Luo R. MicroRNA-379 acts as a tumor suppressor in non-small cell lung cancer by targeting the IGF-1R-mediated AKT and ERK pathways. Oncol Rep. 2017;38:3. doi:10.3892/or.2017.5835

\section{Publish your work in this journal}

Cancer Management and Research is an international, peer-reviewed open access journal focusing on cancer research and the optimal use of preventative and integrated treatment interventions to achieve improved outcomes, enhanced survival and quality of life for the cancer patient.
The manuscript management system is completely online and includes a very quick and fair peer-review system, which is all easy to use. Visit http://www.dovepress.com/testimonials.php to read real quotes from published authors. 